\title{
Parameters Identification of Photovoltaic Cells Based on Differential Evolution Algorithm
}

\author{
Liao Hui ${ }^{1}, 2$, Qiao-Dongkai ${ }^{1}$, Huang-Chong lin ${ }^{1}$, Li-Shi dong ${ }^{1}$ \\ ${ }^{1}$ Guangdong University of Petrochemical Technology, Maoming, Guangdong, 525000 \\ 2 Development center of technology for fruit \& vegetables storage and processing engineering, Maoming, Guangdong, 525000
}

\begin{abstract}
For the complex nonlinear model of photovoltaic cells, traditional evolution strategy is easy to fall into the local optimal and its identification time is too long when taking parameters identification, then the difference algorithm is proposed in this study, which is to solve the problems of parameter identification in photovoltaic cell model, where it is very difficult to achieve with other identification algorithms. In this method, the random data is selected as the initial generation; the successful evolution to the next generation is done through a certain strategy of difference algorithm, which can achieve the effective identification of control parameters. It is proved that the method has a good global optimization and the fast convergence ability, and the simulation results are shown that the differential evolution has high identification ability and it is an effective method to identify the parameters of photovoltaic cells, where the photovoltaic cells can be widely used in other places with these parameters.
\end{abstract}

\section{Introduction}

Photoelectric system is to convert solar energy into electrical energy by means of PV components, due to the reasons of battery materials and preparation technology, the conversion efficiency is low at present. In order to improve the conversion efficiency of solar energy, the characteristics of photovoltaic cells were in-depth study by people [1-5]. When people studied the characteristics of PV cell, they mainly focused on the volt-ampere characteristics of the solar cells output, which can be applied to the control of the maximum power output of battery, these parameters of the battery model including current Iph, reverse saturation current I0, diode quality sub, a series resistance (RS) and the shunt resistance Rsh, through the analysis on battery power input parameters we can control of photovoltaic energy conversion efficiency and battery fault situation in real-time, and then adjust the power to ensure the production smoothly has the very important significance [67].

Parameters of the photovoltaic battery can be measured in the experiment under standard conditions, however, these parameters will change also along with its test condition, so it is necessary to identify the parameters of the photovoltaic cell by use of the I-V relationship, the energy efficiency of the photovoltaic battery can be improved when these parameters taken into the experiment usage[8-10], therefore, the parameters identification of photovoltaic cells are very important, however, the mathematical model of photovoltaic cells is a Lambert $\mathrm{W}$ function, which has a high degree of nonlinear [11]. In recent years, people have tried to describe the optoelectronic system by the derivative method, these data are from the experimental I-V curve [12, 13], under the standard conditions, the parameter values of photovoltaic battery model can be measured through the battery characteristic equation, such as the short-circuit current (ISC), open circuit voltage UOC, the maximum power point of current IM and voltage um and the maximum power value Pm [14]. Due to the effect of the environment working on the parameters of photovoltaic cells, the reference [15] taked into account of the use of conditions when taking identification, and analyzed its impacts on the parameters of the case. it reflects the relationship between the parameters in the steady state of photovoltaic cells and the transient state, the literature [16] have done much research on the case, and then the accuracy of the parameter estimation method were proposed. However, due to the complexity of the nonlinear process of the photovoltaic cell model, when the parameters of this kind of problems are identified, most of the optimization algorithms are easy to fall into the local optimum or the optimization time is too long [17]. 
Through the cooperation and competition among individuals within the group, the differential evolution algorithm (DE) is produced by swarm intelligence to guide the optimization search, retaining the population with global search strategy, which is taking the real coding, a simple mutation operation and the competition strategies, reducing the genetic manipulation of complexity, it has function of memory and dynamic tracking search, which has a strong global convergence ability and robustness by adjusting the searching strategy, the main advantage is that it is not easy to fall into the local optimum, and it has the fast convergence rate [18].

For the above reasons, DE algorithm is proposed in this paper, through the method of selection, crossover and mutation operation, it randomly selected individual, and then evaluating to the better next generation, it can avoid into local optimal in the process of evolution, which ensures the high global optimization success rate, and improving the convergence speed.

\section{De Algorithm}

Assumed to the solved optimization problems has a ndimensional vector, the problem to be solved is composed of the individual $x i=\left(x_{i 1}, x_{i 2}, \cdots, x_{i n}\right)$, a single individual with a random space is a random number of $\left(x_{1 \min }, x_{2 \min }, \cdots x_{i \min }\right)$ and $\left(x_{1 \max }, x_{2 \max }, \cdots x_{i \max }\right)$, its the solution space is [xmin, $x \operatorname{xax}]$, on the basis of a population, in the process of solving, each individual has an evolutionary vector target value.

DE will differ between a generation of individual component vectors evolution crossover and mutation operation, so as to generate a new individual, according to a certain code selection mechanism, decides in the offspring and parent retains, and has been in this selection mechanism evolution until meets the solution conditions setting by the termination.

Differential evolution algorithm is based on the real coded evolutionary algorithm; the overall structure is similar to other evolutionary algorithms, including 3 basic operations of mutation, crossover and selection. Standard differential evolution algorithm mainly includes the following 4 steps.

\subsection{Generating Initial Population}

Assuming that all individuals are in an n-dimensional data set, the data distribution is random, and the randomly generated numbers are expressed as a chromosome.

$$
x_{i j}(0)=\operatorname{radn}_{i j}(0,1)\left(x_{i j}^{U}-x_{i j}^{L}\right)+x_{i j}^{L}
$$

Where $\operatorname{radn}_{i j}(0,1)$ is one of randomly generated numbers, whose scope is $[0,1]$, the upper and lower bounds of the first j chromosome are $x_{i j}^{U}$ and $x_{i j}^{L}$ respectively.

\subsection{Mutation Operation}

The three individuals of $x_{p 1}, x_{p 2}$ and $x_{p 3}$ are randomly selected, the individuals are not the same as each other. That is $i \neq p 1 \neq p 2 \neq p 3$, the basic mutation operator is

$$
h_{i j}(t+1)=x_{p 1 j}(t)+F\left(x_{p 2 j}(t)-x_{p 3 j}(t)\right)
$$

If there is no local optimization problem, the variation operation can be written as

$$
h_{i j}(t+1)=x_{b j}(t)+F\left(x_{p 2 j}(t)-x_{p 3 j}(t)\right)
$$

where, $x_{p 2 j}(t)-x_{p 3 j}(t)$ is the difference of trace, this differential operation is the key of differential evolution algorithm, and $\mathrm{F}$ is the scale variation factor of difference vector. $p 1, p 2, p 3$ are the random integer that represent the number of individuals in the population; $x_{b j}(t)$ is the best individual in the current generation. The first vector on the right side of the equation is called the base vector, which is called the differential vector with $\mathrm{F}$ factor, according to the need to select the appropriate difference vector factor, through the differential evolution algorithm, the evolution population has the best individual information of the current population in the mutation operation, which can improve the convergence rate.

\subsection{Cross Operation}

Crossover operation is to increase the diversity of the population, which can avoid the local optimization in the process of optimization, the algorithm increases the crossover operation, and generates the test vector, and the specific operations are as follows:

$$
v_{i j}(t+1)=\left\{\begin{array}{l}
h_{i j}(t+1), \text { rand } l_{i j} \leq C R \\
x_{i j}(t), \text { rand } l_{i j}>C R
\end{array}\right.
$$

Where, rand $l_{i j}$ is a random function in $[0,1]$, this selection can ensure that the test vectors are different from their corresponding target vectors; $\mathrm{CR}$ is the crossover probability, $C R \in[0,1]$. 


\subsection{Select Operation}

In the setting of search space, the algorithm will be the mutation and crossover operations on the basis of the generation of the initial population, in the corresponding search space, the algorithm will judge the test vector of each generation whether beyond its upper generation or not, which will be the initialization with a uniform random number when it beyond the searching space of the corresponding dimension, and the greedy selection method of operation will avoid the premature convergence in certain procedures, and then it will get better in the next generation of individuals.

In order to determine whether $x_{i}(t)$ is a member of the next generation, the test vector $v_{i j}(t+1)$ and the target vector $v_{i j}(t)$ are compared to the evaluation function:

$$
x_{i}(t+1)\left\{\begin{array}{l}
v_{i}(t+1), f\left(v_{i 1}(t+1), \cdots, v_{i n}(t+1)\right)<f\left(v_{i 1}(t), \cdots, v_{i n}(t)\right) \\
x_{i j}(t), f\left(v_{i 1}(t+1), \cdots, v_{i n}(t+1)\right) \geq f\left(v_{i 1}(t), \cdots, v_{i n}(t)\right)
\end{array}\right.
$$

Repeat steps (2) to step (4), the final generation will be the best generation until the maximum number of iterations to reach $\mathrm{G}$.

\section{Identification of Photovoltaic Cells Model Parameters}

The photovoltaic cell is a circuit that converts light energy into electric energy by photovoltaic panels, which can be equivalent to the circuit shown in Fig.1. The expression of the current and voltage characteristics in the circuit can be expressed as Rp model.

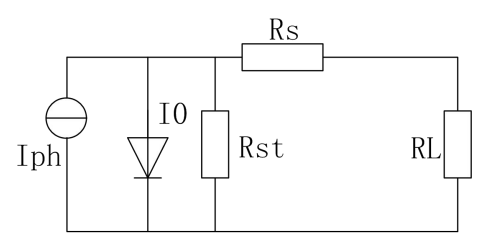

Fig. 1 Equivalent Circuit of Photovoltaic Cell

$$
I=I_{p h}-I_{0}\left\{\exp \frac{q\left(V+I R_{s}\right)}{K A T}-1\right\}-\frac{V+I R_{s}}{R_{s h}}
$$

Where :

$\mathrm{V}$ - load voltage at both ends;

I - by load current;

Iph - photo induced current;

IO - diode reverse saturation current;

A - diode quality factor;

Rs - battery series resistance;

Rsh - battery parallel resistance;

$\mathrm{T}$ - absolute temperature of the battery;

$\mathrm{K}$ - Pohl Seidman constant (1.380e-23 J/K);
Q - electron charge (1.608e-19 DEG C).

\subsection{Establishment of Parameter Identification Model}

Photovoltaic cell model is a highly nonlinear equation; differential evolution algorithm will establish the fitness function by the use of the explicit expression of the current, and then converting the PV model into an explicit expression. According to the characteristics of photovoltaic cells, it can be transformed to the transcendental function of $\mathrm{Y}(\mathrm{x})=\mathrm{XeX}$. Lambert $\mathrm{W}$ function which can be used to solve the current voltage equation, the solution of the form is $\mathrm{W}(\mathrm{Y}(\mathrm{x}))$, the differential evolution algorithm can be used to identify the parameters of the photovoltaic cell model.

Depending on the upper type, the current and voltage of the photovoltaic cell are converted to an explicit equation that can be obtained.

$$
I=\frac{R_{s h}\left(I_{p h}+I_{0}\right)-V}{R_{s h}+R_{s}}-\frac{A V_{t h}}{R_{s}} W\left(\frac{R_{s h} R_{s} I_{0}}{\left(R_{s h}+R_{s}\right) A V_{t h}} \exp \left(\frac{R_{s h} V+R_{s h} R_{s}\left(I_{p h}+I_{0}\right)}{A V_{t h}\left(R_{s h}+R_{s}\right)}\right)\right)
$$

Tab. 1 shows the parameters under standard conditions $\left(25^{\circ} \mathrm{C}\right.$ $1000 \mathrm{~W} / \mathrm{m} 2$,) of SFM50-18 photovoltaic cell panel. Fig. 2 is the I$\mathrm{V}$ curves under different illumination conditions.

Tab.1 Parameters of Photovoltaic Cell Panel

\begin{tabular}{llll}
\hline Parameter & $\begin{array}{l}\text { parameter } \\
\text { value }\end{array}$ & Parameter & $\begin{array}{l}\text { parameter } \\
\text { value }\end{array}$ \\
\hline Imp & $2.9 \mathrm{~A}$ & Voc & $21.5 \mathrm{~V}$ \\
Vmp & $17.3 \mathrm{~V}$ & Pmax,e & $50 \mathrm{~W}$ \\
Isc & $3.18 \mathrm{~A}$ & Ns & 36 \\
\hline
\end{tabular}

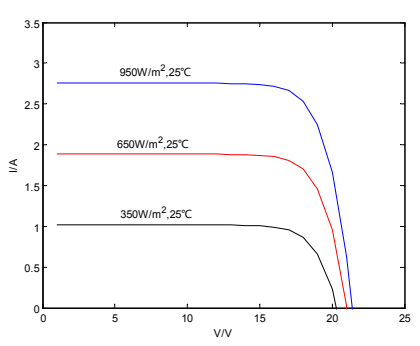

Fig.2 The Change Of Current-Voltage Under Different Light Intensity

\subsection{Parameter Identification}

After the conversion of the explicit expression of the photovoltaic cell model, the 5 parameters of the photovoltaic cell current-voltage model are identified by using DE. Objective function value change reflects the algorithm to identify the effect of the model; fig.2 shows the parameters of the DE algorithm to identify the value of the objective function. It can be seen from the figure that the parameter identification can be fast and stable in a numerical value, for the PV cell voltage model parameters identification, DE algorithm has a better stability. 
For different size of the population, the speed of the algorithm is not consistent, the smaller the size of the population received faster. Fig. 3 is the comparison of the size of the 3 different populations, From which it can be calculated, in fact, when improving the identification of convergence rate, the smaller the size of the population is suitable, however, the small size of the population will induce accuracy of parameter identification. Table 3 is a comparison of the results of the identification of 3 different species.

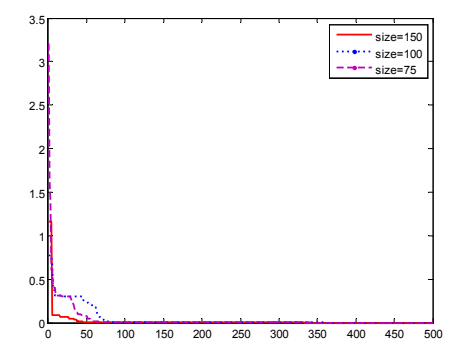

Fig.3 Convergence of Parameter Identification under Different Population

When the condition is changed, the parameter values of the photovoltaic cell will be changed. Therefore, changing the intensity of the light will be identified, the parameter identification results are shown in Tab.2 under different light intensity conditions, the results show that, the identification parameters were changed along with the test conditions.

Tab.2 Parameters of the Battery Model for De Identification When the Light Intensity Changes

\begin{tabular}{llllll}
\hline $\begin{array}{l}\text { light } \\
\text { intensity } \\
(\mathrm{W} / \mathrm{m} 2)\end{array}$ & $I \mathrm{ph}$ & $I \mathrm{o} / \mathrm{A}$ & $A$ & $R \mathrm{~s} / \Omega$ & $R \mathrm{sh} / \Omega$ \\
\hline 700 & 2.836 & 8.201 & 1.420 & 0.027 & 203.36 \\
600 & 2.421 & 8.182 & 1.428 & 0.029 & 214.66 \\
500 & 2.105 & 8.168 & 1.536 & 0.031 & 249.50 \\
400 & 1.666 & 8.147 & 1.471 & 0.034 & 289.71 \\
300 & 1.258 & 8.126 & 1.513 & 0.036 & 311.65 \\
200 & 0.978 & 8.106 & 1.505 & 0.039 & 379.89 \\
100 & 0.685 & 8.085 & 1.498 & 0.041 & 440.89 \\
\hline
\end{tabular}

The data of identification and the actual data were applied in the volt-ampere circuit test, fig. 4 was the results of simulation experiments, both capitals of error is very small that the parameter identification results can be applied to the actual current-voltage in the model.

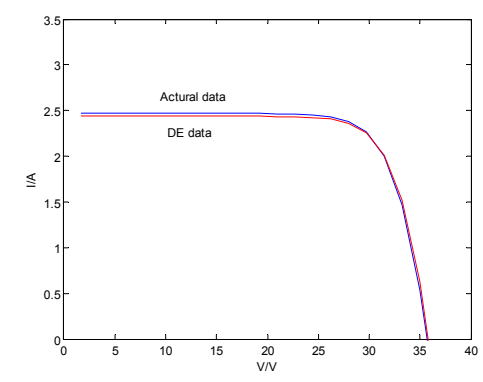

Fig.4 Identification results and Measured Conditions, the IV Curve of Photovoltaic Cells

\section{Conclusions}

In view of the problems is difficult to determine the parameters of the photovoltaic battery, this paper presents a differential evolution algorithm, and the corresponding research is carried out, for example, the parameters of differential evolution algorithm will quickly and accurately identify the parameters of the photovoltaic cell when it set reasonably, such as population size, the identification results showed that, after a certain time of calculation, the DE algorithm can take identification for photovoltaic battery model parameters accurately, which were close to the actual value of the optimal solution, and proved that the algorithm has higher stability and precision of identification; at the same time, for different population sizes, the algorithm also have global optimization and rapid convergence ability, and in fact, the speed and accuracy of identification process have a certain impact on the convergence, as to how to choose a reasonable initial value, which can reach a higher accuracy under the random initial value, and the system fault can be online identification is the key direction of the next step work.

\section{Acknowledgement}

This paper is supported by the science and technology project of Guangdong province (2014A010106033); the natural science foundation of Guangdong Province, the startup project of Guangdong province (2014A030310376); The project of young innovative talents in Colleges and universities of Guangdong province (631051)

\section{References}

1. Fayrouz Dkhichi; Benyounes Oukarfi; Youssef El Kouari; David Ouoba; Abderrahim Fakkar. Neural network based integration of MPPT and diagnosis of degradation for photovoltaic module[J]. Optical and Quantum Electronics, 2016,48(2)

2. SACHIN VRAJLAL RAJANI; VIVEK J PANDYA. Simulation and comparison of perturb and observe and 
incremental conductance MPPT algorithms for solar energy system connected to grid[J]. Sadhana, 2015, Vol.40 (1):139-153.

3. Mahdi Rajabi Vincheh; Abbas Kargar; Gholamreza Arab Markadeh. Arabian A Hybrid Control Method for Maximum Power Point Tracking (MPPT) in Photovoltaic Systems[J].Journal for Science and Engineering. Section A: Sciences. 2014,39(6):47154725 .

4. Hua Zhu; Zhang-lu Xu; Zi-juan Cao. Experimental research on charging characteristics of a solar photovoltaic system by the pressure-control method[J]. Journal of Zhejiang University. Science, 2011,12(6):470-474.

5. Ismail Nakir;Ali Durusu;Hakan Akca;Ali Ajder;Ramazan Ayaz;Enes Ugur;Mugdesem Tanrioven. A New MPPT Algorithm for Vehicle Integrated Solar Energy System[J]. Journal of Energy Resources Technology, 2015,138(2):1034-1038.

6. Nezihe Yildirana; Emin Tacerb. Identification of photovoltaic cell single diode discrete model parameters based on datasheet values[J]. Solar Energy, 2016,(127):175-183.

7. Dani Rusirawana; István Farkasb. Identification of Model Parameters of the Photovoltaic Solar Cells[J]. Energy Procedia, 2014 (57): 39-46.

8. LIU Fangrui, DUAN Shanxu, LIU Fei, et al. A variable step size INC MPPT method for PV systems[J]. IEEE Transactions on Industrial Electronics, 2008, 55(7): 2622-2628.

9. PATEL H, AGARWAL V. Maximum power point tracking scheme for PV systems operating under partially shaded condition $[\mathrm{J}]$. IEEE Transactions on Industrial Electronics, 2008, 55(4):1689-1698.

10. KOTTAS T L, BOUTALIS Y S, KARLIS A D. New maximum power point tracker for PV arrays using fuzzy controller in close cooperation with fuzzy cognitive networks[J].. IEEE Transactions on Energy Conversion, 2006, 21(3): 793-803.

11. Ghani, F.;Duke, M.;Carson, J. Numerical calculation of series and shunt resistance of a photovoltaic cell using theLambert W-function: Experimental evaluation[J]. Solar Energy, 2013(87):246-253.

12. Yadir S, Benhmida M, Sidki M, et al. New method for extracting the model physical parameters of solar cells using explicit analytic solutions of current-voltage equation[C]. International Conference on icroelectronics(ICM), 2009: 390-393.

13. Lyden S, Haque M E, Gargoom A. et al. Modelling and parameter estimation of photovoltaic cell[C]. Universities Power Engineering Conference (AUPEC), 2012: 1-6.

14. Zhou Jianliang, Wang Bing, Zhang Yiming. Parameter identification and output power prediction of photovoltaic array based on the measured data[J]. Renewable Energy Resources, 2012, 30(7): 1-4.
15. Lyden S, Haque M E, Gargoom A. et al. Modelling and parameter estimation of photovoltaic cell[C]. Universities Power Engineering Conference (AUPEC), 2012: 1-6.

16. Neukom M T, Zufle S, Ruhstaller B. Reliable extraction of organic solar cell parameters by combining steady-state and transient techniques[J]. Organic Electronics, 2012, 13(12): 2910-2916.

17. Cheng Ze Dong Mengnan Yang Tiankai Han Lijie. Extraction of Solar Cell Model Parameters Based on Self-Adaptive Chaos Particle Swarm Optimization Algorithm[J]. TRANSACTIONS OF CHINA ELECTROTECHNICAL SOCIETY, 2014, 29(9): 245252.

18. JIAN Xian-zhong, YAN Jun, FAN Jian-pen, HOU Yixin1, GUO Qiang. MPPT Control Method of Photovoltaic Array Based on Differential Evolution Algorithm[J]. Control Engineering of China, 2014, 21(4):559-556. 